\title{
Flexibiliser par la procédure
}

Les réformes divergentes des licenciements économiques en France et en Italie

Procedural flexibilization: diverging reforms of economic redundancies in France and Italy

\section{Raphaël Dalmasso}

\section{(2) OpenEdition}

\section{Journals}

Édition électronique

URL : http://journals.openedition.org/travailemploi/6631

DOI : 10.4000/travailemploi.6631

ISSN : 1775-416X

Éditeur

DARES - Ministère du Travail

Édition imprimée

Date de publication : 1 avril 2015

Pagination : 55-67

ISSN : 0224-4365

Référence électronique

Raphaël Dalmasso, «Flexibiliser par la procédure », Travail et Emploi [En ligne], 142 | avril-juin 2015, mis en ligne le 01 avril 2017, consulté le 03 mai 2019. URL : http://journals.openedition.org/

travailemploi/6631; DOI : 10.4000/travailemploi.6631 


\title{
Flexibiliser par la procédure : les réformes divergentes des licenciements économiques en France et en Italie
}

\author{
Raphaël Dalmasso(*)
}

Le droit du licenciement économique a récemment fait l'objet, en France comme en Italie, d'une profonde réforme. Si ces modifications ont partagé un objectif commun de flexibiliser et sécuriser les ruptures, leur contenu est cependant très différent. En effet, à travers deux réformes procédurales, les deux législations assignent au juge et au contentieux des rôles différents, voire opposés. Ce faisant, elles démontrent que l'objectif de flexibilité et de sécurisation ne saurait, en droit du licenciement économique, avoir de signification juridique univoque.

Depuis une quarantaine d'années, aux mêmes périodes environ, la France et l'Italie ont procédé à plusieurs réformes du droit du licenciement, particulièrement du licenciement économique. Ainsi, la loi française de $1973^{(1)}$ relative à la procédure des licenciements se nourrissait des réformes italiennes de $1966^{(2)}$ sur les licenciements pour justes motifs subjectifs et pour justes motifs objectifs, et du statut des travailleurs de $1970^{(3)}$. De même, en $1975^{(4)}$, les deux droits évoluaient de manière plus ou moins marquée sous l'influence du droit communautaire. Enfin, à la fin des années 1980 et au début des années 1990, pendant que la France modifiait en $1989^{(5)}$ sa procédure de licenciement économique avec la création du plan social, la procédure de

(*) Université de Lorraine, chercheur associé au Centre d'études de l'emploi (CEE), membre de l'Institut FrançoisGény; Raphael.dalmasso@univ-lorraine.fr

(1) Loi $n^{\circ}$ 73-680 du 13 juillet 1973, modifiant la procédure des licenciements, prévoyant notamment les étapes préalables à l'envoi de la lettre de licenciement et la nécessité de motiver la rupture.

(2) Loi n 604/1966 du 15 juillet 1966 prévoyant la nécessité de motiver les licenciements.

(3) Loi n 300/1970 du 14 mai 1970, communément appelée «statut des travailleurs». Cette loi a notamment organisé, dans son article 18 , les régimes d'indemnisation des salariés en cas de licenciement illégitime.

(4) Directive $n^{\circ} 75 / 129 / \mathrm{CE}$ du 17 février 1975 imposant l'instauration d'une procédure spécifique aux licenciements économiques collectifs. La loi française du 3 janvier 1975 crée un régime juridique propre aux licenciements économiques. Du côté italien, le législateur a estimé, à tort, que la loi de 1966 était conforme à la directive de 1975 , et a adapté a minima son droit, uniquement en matière de chômage partiel, dans la loi $\mathrm{n}^{\circ} 164 / 1975$ du 20 mai 1975. Après deux condamnations par la Cour de justice des communautés européennes (CJCE, 8 juin 1982, affaire 91/81; CJCE 6 novembre 1985, affaire 131/84), l'Italie a pleinement transposé la directive par la loi $\mathrm{n}^{\circ} 223 / 1991$ du 23 juillet 1991 .

(5) Loi n 89-549 du 2 août 1989 relative à la prévention du licenciement économique et au droit à la conversion. licenciement économique italienne était totalement refondée dans une loi de $1991^{(6)}$.

En $2012^{(7)}$ pour l'Italie et $2013^{(8)}$ pour la France, les droits des licenciements économiques ont été de nouveau fortement réformés. Malgré de notables nuances, le but de ces réformes est comparable : il s'agit de rendre plus flexibles et plus sûrs, principalement pour les employeurs, les licenciements pour motif économique (I). Cependant, si, au sein de ces réformes, on choisit de prendre en considération la place dévolue au contentieux et au pouvoir de contrôle du juge, un constat s'impose : les deux pays empruntent des voies procédurales différentes, voire opposées. Cela indique que la flexibilisation juridique du contrat de travail peut prendre plusieurs directions. Cette pluralité s'explique par la différence de cible visée : un acteur, le juge, pour le droit français, est suspecté de trop contrôler les procédures de licenciement; un texte, l'article $18 \mathrm{du}$ statut des travailleurs, pour le droit italien, est jugé trop favorable aux salariés (II). Ces deux réformes de flexibilisation des licenciements économiques risquent cependant, dans les deux pays, de conduire à des résultats mitigés : il n'est pas certain que le contentieux ou le contrôle judiciaire soit moins important à l'avenir en France, tandis qu'en Italie, la réforme de 2012 a d'ores et déjà été remise en cause depuis la fin de l'année 2014(9) (III).

(6) Loi n 223/1991 précitée.

(7) Loi $n^{\circ} 92 / 2012$ du 28 juin 2012 relative à réforme du marché du travail dans une perspective de croissance.

(8) Loi $\mathrm{n}^{\circ}$ 2013-504 du 14 juin 2013 relative à la sécurisation de l'emploi, traduction de l'accord national interprofessionnel (ANI) du 11 janvier 2013 pour un nouveau modèle économique et social au service de la compétitivité des entreprises et de la sécurisation de l'emploi et des parcours professionnels des salariés.

(9) Loi n ${ }^{\circ}$ 183/2014 du 10 décembre 2014 (Jobs Act); décret de la loi $\mathrm{n}^{\circ} 23 / 2015 \mathrm{du} 4$ mars 2015. 


\section{Une flexibilisation par la procédure}

La réforme récente du droit des licenciements économiques ne s'est pas imposée de la même manière en France et en Italie. En Italie, c'est sous la pression d'une situation économique sérieusement dégradée et des fortes incitations de la Banque centrale européenne que le gouvernement Berlusconi, puis le gouvernement Monti, ont modifié le droit du travail dans une direction assumée de flexibilité. En France, les motivations de la réforme demeurent, encore aujourd'hui, plus ambiguës. Cependant, dans les deux pays, le législateur a choisi de ne pas modifier la définition des licenciements économiques, mais de se centrer sur des modifications procédurales. Ces évolutions techniques changent profondément la compréhension et l'usage du droit du licenciement économique.

\section{Deux réformes d'une ampleur différente}

La réforme italienne de 2012 et la réforme française de 2013 ont un champ juridique d'application très différent. Nous n'aborderons ici que les procédures du licenciement pour motif économique. Mentionnons cependant, sans les détailler, les autres aspects.

En Italie, la loi de $2012^{(10)}$ en matière de réforme du marché du travail concerne principalement ${ }^{(11)}$ le droit du licenciement, et sa procédure ${ }^{(12)}$. À la différence du droit français, ces modifications ne portent pas sur les seuls licenciements économiques, puisqu'elles touchent aussi les licenciements pour motif personnel et les licenciements discriminatoires. La réforme du marché du travail a donc été conçue comme une réforme globale du licenciement.

En France, la loi de sécurisation de l'emploi du 4 mai 2013, traduction assez fidèle de l'accord national interprofessionnel du 11 janvier 2013, vise également à réformer le marché du travail. Le texte a cependant un objet différent du texte italien. Outre les licenciements économiques, les modifications législatives portent sur une multitude de thèmes : la formation professionnelle, le temps partiel, l'activité partielle, le développement d'accords «de maintien de l'emploi», les droits rechargeables à l'assurance-chômage, la complémentaire santé, etc. Le licenciement économique et sa procédure ne sont donc que l'un des aspects de la réforme de 2013, même si, pour beaucoup de commentateurs (FABRE, 2013 par exemple), il s'agit là des modifications les

(10) Loi n 92/2012 du 28 juin 2012, précitée.

(11) La loi apporte également des changements importants dans la définition de certains contrats de travail spéciaux; elle modifie en outre le régime de chômage partiel et étend celui de l'assurance-chômage à l'ensemble des salariés.

(12) La réforme de 2012 a été complétée et précisée par un «decreto lavoro» («décret travail») n 34 du 20 mars 2014, notamment sur les contrats à durée déterminée. plus importantes et les plus attendues. En revanche, les licenciements personnels ne sont pas modifiés.

Dans les deux droits, ne prendre en considération que les modifications relatives au licenciement économique pourrait apparaître comme réducteur car ne permettant pas de comprendre l'économie générale de chaque dispositif, faite de compromis inédits. Cependant, dans une optique de comparaison des droits, il est nécessaire de mettre en perspective un même objet d'étude, ici le droit du licenciement économique, dont la procédure est conjointement modifiée. La comparaison menée ici se référera à la méthode des équivalents fonctionnels présentée par Konrad ZweIGERT et Hein Kötz (1998). Ces auteurs partent du principe que si les systèmes juridiques modernes sont confrontés aux mêmes problèmes, ils peuvent les résoudre avec des procédés, des types de normes, ou même des catégories juridiques différentes ${ }^{(13)}$. Il convient donc de comparer les droits en partant d'une ou plusieurs fonctions (principe de fonctionnalité) pour prendre en considération toutes les normes de chaque ordonnancement relatives à ces fonctions. Le droit français fait référence à la notion de licenciement pour motif économique, tandis que le droit italien évoque les licenciements pour juste motif objectif(14) et les licenciements collectifs pour réduction de personnel ${ }^{(15)}$. La catégorie juridique «licenciement économique» n'est donc pas construite de la même manière en France et en Italie (Dalmasso, 2009), mais les deux droits ont bien un corpus de textes relatifs à la même fonction : encadrer les licenciements économiques.

\section{Italie : une flexibilisation assumée}

Une grande partie du droit italien des licenciements n'est pas codifiée, et résulte de lois éparses, souvent anciennes ${ }^{(16)}$. Comme nous l'avons mentionné plus haut, les trois principales lois concernant notre objet sont celles de 1966, 1970 et 1991.

La loi $\mathrm{n}^{\circ}$ 604/1966 du 15 juillet 1966 donne une définition du licenciement individuel. Son

(13) Voir aussi, pour une démarche assez similaire, DAVID (1950, pp. 10-11) : «Le comparatiste - j'entends par là le juriste qui veut faire usage de la méthode comparative - doit donc éviter des erreurs. Il doit connaître la théorie des sources du droit admise par les juristes du pays dont il entreprend d'étudier le droit; il doit étudier le droit étranger comme ferait le juriste étranger. Les principes qui doivent le guider [...] sont ceux qui doivent guider l'historien du droit. [...] Le comparatiste doit se délivrer de la tendance naturelle qu'il a, juriste français, à faire ses recherches de droit étranger selon les méthodes qu'il applique dans ses recherches de droit français. Il ne doit pas considérer comme ayant une valeur universelle les procédés techniques de recherche et d'interprétation auxquels il est habitué. Agir autrement, c'est vouloir ouvrir toutes les serrures avec une même clef.»

(14) Loi n 604/1966 du 15 juillet 1966, précitée.

(15) Loi n 223/1991 du 23 juillet 1991, précitée.

(16) Il n'y a pas de code du travail en Italie, et le Codice civile est très lacunaire en droit du travail. 
article 1 er(17) dispose que, pour être valide, un licenciement doit être justifié par l'employeur. D'après cet article, l'exigence de motivation selon que la rupture est à l'initiative du salarié ou à celle de l'employeur n'est pas la même : le salarié souhaitant rompre son contrat à durée indéterminée (CDI) n'a en effet pas à le justifier. L'article 3 propose une définition du «juste motif» de licenciement : «Le licenciement pour motif justifié avec préavis est déterminé par une notable inexécution des obligations résultant du contrat de travail de la part du salarié, ou bien de raisons inhérentes à l'activité productive, à l'organisation du travail et à son fonctionnement régulier. » Cet article prévoit ainsi deux types de licenciements. Par souci de simplification, les auteurs italiens évoquent le plus souvent, dans le premier cas, le licenciement «pour juste motif subjectif» et, dans le second cas, le licenciement «pour juste motif objectif». Cette pratique remonte à la création de la loi si bien que la distinction entre licenciements «objectifs» et «subjectifs» semble aujourd'hui communément admise. Ce juste motif «objectif» de licenciement peut être assimilé, à quelques nuances près (DAlmasso, 2009, pp. 42 et suiv.; pp. 203 et suiv.), au licenciement pour motif économique individuel français.

À la différence du droit français, qui ne donne qu'une définition du licenciement économique (18), qu'il soit individuel ou collectif, le droit italien fournit une définition spécifique du licenciement économique collectif dans 1'article 24 de la loi $\mathrm{n}^{\circ} 223 / 1991 \mathrm{du} 23$ juillet 1991. Cet article indique que «les dispositions prévues à l'article 4, alinéa 2 à 12 et 15 bis, et à l'article 5, alinéa 1 à 5 (19), s'appliquent à toute entreprise employant plus de quinze salariés qui, suite à une réduction ou une transformation d'activité ou de travail, envisage d'effectuer au moins cinq licenciements dans une période de cent vingt jours dans le même établissement ou dans le cadre de plusieurs établissements appartenant à la même province. Ces dispositions trouvent application pour tout licenciement qui, dans la même période et dans le même cadre, peut être considéré comme la conséquence de la même réduction ou transformation. Les dispositions du

(17) «Nel rapporto di lavoro a tempo indeterminato, intercedente con datori di lavoro privati o con enti pubblici, ove la stabilità non sia assicurata da norme di legge, di regolamento e di contratto collectivo o individuale, il licenziamento non puo avvenire che per giusta causa ai sensi dell'articolo 2119 del Codice civile o per giustificato motivo "; "dans les contrats de travail à durée indéterminée, signés avec des employeurs privés ou des collectivités publiques où la stabilité contractuelle n'est pas assurée par une loi, un règlement, ou un contrat collectif ou individuel, le licenciement du salarié ne peut intervenir qu'en cas de juste cause au sens de l'article 2119 du Code civil ou pour un motif justifié» (toutes les traductions italiennes de ce document sont de l'auteur).

(18) Art. L. 1233-3, Code du travail.

(19) Il s'agit de la procédure de consultation syndicale, obligatoire pour les licenciements collectifs. premier alinéa s'appliquent également lorsque l'employeur entend cesser son activité(20)». Il s'agit donc de licenciements collectifs résultant soit d'une réduction ou d'une transformation d'activité ou de travail, soit d'une cessation d'activité de l'entreprise.

Le droit italien du licenciement est enfin réglementé par le «statut des travailleurs», défini par la loi $n^{\circ} 300 / 1970$ du 14 mai 1970. Ce texte est particulièrement important, car c'est lui qui a fixé, dans son article 18, sous certaines hypothèses, le principe de la réintégration du salarié licencié de manière illégitime. À partir des années 1990, il cristallise le débat sur la flexibilité des licenciements. Au cours des vingt dernières années, des réformes du licenciement économique et de son régime indemnitaire ont à plusieurs reprises été souhaitées par les gouvernements, puis reportées ou annulées sine die. En 2001, notamment, un livre blanc présenté par le ministre du Travail Roberto Maroni, suivi d'un projet de loi ${ }^{(21)}$, entend réformer l'article 18 du statut des travailleurs en vue de mettre fin à la réintégration des salariés licenciés. Mais une forte opposition syndicale contraint le législateur à abandonner la réforme.

Au cours de l'été 2011, l'Italie traverse une crise économique très profonde, le pays se trouvant au bord du défaut de paiement. Le 5 août 2011, la Banque centrale européenne (BCE) fait parvenir au gouvernement italien de Silvio Berlusconi une lettre signée par Jean-Claude Trichet et Mario Draghi (22) l'enjoignant de modifier sans délai «les normes réglementant l'embauche et le licenciement» dans le sens d'une plus grande flexibilité. La lettre, censée rester confidentielle, a été publiée dans le Corriere della Sera au cours du mois de septembre 2011. Après la chute du gouvernement Berlusconi, le gouvernement Monti annonce que des évolutions doivent avoir lieu en droit du travail. Le projet de réforme du marché du travail est alors porté par la ministre Elsa Fornero, qui consulte rapidement les organisations syndicales. Menée au pas de charge, la réforme Fornero du marché du travail se concrétise par la loi $\mathrm{n}^{\circ}$ 92/2012 du 28 juin 2012 intitulée «Disposizioni in materia di riforma del mercato del

(20) «Le disposizioni di cui all'articolo 4, commi da 2 a 12 e 15bis, e all'articolo 5, commi da 1 a 5, si applicano alle imprese che occupano più di quindici dipendenti e che, in conseguenza di una riduzione o trasformazione di attività o di lavoro, intendano effettuare almeno cinque licenziamenti, nel'arco di centoventi giorni, in ciascuna unità produttiva, o in più unità produttive nell'ambito del territorio di una stessa provincia. Tali disposizioni si applicano per tutti i licenziamenti che, nello stesso arco di tempo e nello stesso ambito, siano comunque riconducibili alla medesima riduzione o trasformazione. Le disposizione richiamate nel c. $1^{\circ}$ si applicano anche quando le imprese di cui al medesimo comma intendano cessare l'attività." "

(21) Projet de loi $n^{\circ} 848-\mathrm{S}$.

(22) Présidents successifs de la BCE en 2011. 
lavoro in una prospettiva di crescita ${ }^{(23)} 》$. L'article 1 er (24) de cette loi annonce la philosophie générale du texte, à savoir la mise en place d'un marché du travail plus inclusif et dynamique. Le terme «inclusif» indique que le législateur souhaite favoriser l'insertion dans le monde du travail de toutes les populations exclues, tandis que le terme «dynamique» fait référence aux blocages constatés dans le droit du travail. Plus précisément, la loi vise à redistribuer plus équitablement les protections en matière d'emploi: favoriser les embauches des travailleurs exclus, mais aussi et surtout, revoir les règles protectrices des licenciements des salariés. Le législateur ne s'en est donc pas caché : cette loi a pour objectif de faciliter les ruptures, ou de flexibiliser le contrat de travail (Speziale, 2012). La voie choisie est celle d'une réforme procédurale du licenciement économique. Les lois de 1966 et 1991, qui définissent le licenciement économique, ne sont pas modifiées; seul est impacté le statut des travailleurs de 1970.

\section{France : une "sécurisation" à la signification controversée}

En France également, les partenaires sociaux, puis le législateur ont décidé de ne pas modifier la définition légale des licenciements économiques figurant à l'article L. 1233-3 du Code du travail. En 2013, c'est la procédure de licenciement économique collectif qui est profondément remaniée.

En France, l'interprétation donnée à la réforme varie encore considérablement aujourd'hui selon les acteurs des négociations. Comme nous allons le voir, pour la CFDT ${ }^{(25)}$, la réforme entière, y compris ses dispositions concernant les licenciements économiques, est favorable à la fois aux employeurs et

(23) «Dispositions en matière de réforme du marché du travail dans une perspective de croissance » (traduction de l'auteur); pour deux présentations du texte, voir VIDIRI (2012) et Scognamiglio (2012).

(24) «La presente legge dispone misure e interventi intesi a realizzare un mercato del lavoro inclusivo e dinamico, in grado di contribuire alla creazione di occupazione, in quantita' $e$ qualita', alla crescita sociale ed economica e alla riduzione permanente del tasso di disoccupazione, in particolare [...] ridistribuendo in modo piu' equo le tutele dell'impiego, da un lato contrastando l'uso improprio e strumentale degli elementi di flessibilita' progressivamente introdotti nell'ordinamento con riguardo alle tipologie contrattuali; dall'altro adeguando contestualmente alle esigenze del mutato contesto di riferimento la disciplina del licenziamento, con previsione altresi' di un procedimento giudiziario specifico per accelerare la definizione delle relative controversie." Traduction résumée de l'auteur: «La présente loi fixe des mesures destinées à mettre en place un marché du travail inclusif et dynamique, afin de contribuer à la création d'emplois en quantité et de qualité, à la croissance sociale et économique et à la réduction permanente du taux de chômage, en particulier en redistribuant de manière plus équitable les protections de l'emploi, d'un côté, en luttant contre un usage impropre de certaines flexibilités liées aux types de contrats, de l'autre, en adaptant la procédure du licenciement pour améliorer et accélérer la résolution des litiges. » (25) Confédération française démocratique du travail. aux salariés. Pour le Medef ${ }^{(26)}$ et certains syndicats non signataires ${ }^{(27)}$ de l'Accord national interprofessionnel du 11 janvier 2013 en revanche, ce dernier comprend bien deux volets distincts avec, d'une part, des éléments protecteurs pour les salariés et, d'autre part et surtout, une forme de flexibilisation des contrats qui bénéficie aux employeurs via la réforme de la procédure des licenciements économiques.

Un des principaux négociateurs de l'accord pour le compte du Medef indique ainsi que «le sens de l'accord tient en un mot : compétitivité» (Foucher, 2013, p. 89). Mais, selon lui, l'accord doit se comprendre en distinguant les mesures favorables aux salariés de celles favorables aux employeurs. $\mathrm{Au}$ bénéfice des salariés, l'auteur cite notamment la couverture complémentaire santé collective, les droits rechargeables à l'assurance-chômage et le compte personnel de formation (Foucher, 2013, p. 91). Côté employeurs, il reconnaît que «jamais non plus un accord ou une loi n'avait apporté autant de souplesse nouvelle» et recense par exemple les accords de mobilité interne, ceux de maintien dans l'emploi, la réforme du chômage partiel, la mise en place de délais préfix ${ }^{(28)}$, la réforme de la conciliation prud'homale, ou les nouvelles règles encadrant les plans de sauvegarde de l'emploi (Foucher, 2013, p. 92). Pour toutes les dispositions liées au droit du licenciement économique, l'auteur admet qu'elles correspondent aux demandes des employeurs et que la nouvelle norme facilite les licenciements économiques en les flexibilisant.

Cette lecture de l'accord en termes de concessions réciproques est partagée par un négociateur du syndicat non signataire Force ouvrière. Le déséquilibre trop important entre les concessions accordées aux salariés et celles consenties aux employeurs a conduit ce syndicat à refuser de le signer. Le négociateur estime notamment que le droit des licenciements économiques se trouve trop flexibilisé : «sous couvert de l'accord collectif majoritaire, l'accord national interprofessionnel fragilise des droits individuels des salariés» (LARDY, 2013, p. 103).

(26) Mouvement des entreprises de France.

(27) Cet accord national interprofessionnel a été signé, pour les syndicats de salariés, par la CFDT (Confédération française démocratique du travail), la CFTC (Confédération française des travailleurs chrétiens) et la CFE-CGC (Confédération française de l'encadrement-Confédération générale des cadres), et du côté des employeurs, par le Medef, la CGPME (Confédération générale des petites et moyennes entreprises) et l'UPA (Union professionnelle artisanale). À l'inverse, la CGT (Confédération générale du travail) et la CGT-FO (Force ouvrière) n'ont pas signé l'accord.

(28) En procédure civile, il s'agit d'un «délai dont la méconnaissance constitue une fin de non-recevoir, entrainnant la perte du droit d'agir en justice. Dans ce cas, le tribunal juge, sans examen au fond, que la demande est irrecevable.» (Source : Braudo S. (1996-2015), Dictionnaire du droit privé français; définition disponible en ligne à l'adresse http://www. dictionnaire-juridique.com/definition/prefix.php; consultée le 26 août 2015.) 
À l'inverse, dans le magazine de la CFDT, Aurélie SeIGNe et Emmanuelle PIRAT partent du constat que le marché du travail français est gravement malade, et qu'il est devenu de facto très flexible, notamment par l'usage systématisé des contrats précaires. Les auteures précisent donc que «c'est en priorité à cette réalité que la CFDT a voulu s'attaquer. En changer la donne pour ceux qui portent l'hyperflexibilité non avouée du marché du travail. La CFDT a ferraillé durant les trois mois de négociation face au patronat afin d'obtenir satisfaction sur ses quatre exigences incontournables : la taxation des contrats courts, la limitation des temps partiels subis, des droits rechargeables pour les demandeurs d'emploi, la généralisation à tous les salariés de la complémentaire santé» (SEIGNE, PIRAT, 2013, p. 14). Les auteures indiquent cependant également que «l'accord signé [...] revoit les procédures de licenciement collectif pour éviter les aberrations comme l'annulation de plans sociaux des années après que les salariés ont été licenciés [...]. Désormais, la procédure et le contenu du plan de sauvegarde de l'emploi seront soit négociés par accord majoritaire, soit contrôlés par l'administration. Pour la CFDT, c'est une sécurité supplémentaire apportée tant aux salariés qu'à l'entreprise » (Seigne, Pirat, 2013, p. 16).

À l'image des partenaires sociaux, la doctrine juridique est divisée. Certains estiment que la réforme a comme ambition principale de faciliter les licenciements collectifs (BONNECHÈre, 2013; GÉA, 2013; FABRE, 2013; Couturier, 2013), tandis que d'autres auteurs s'interrogent sur la réalité pratique de cette flexibilité (Jolivet, 2013; TARASEWICz, 2013).

En résumé, si tout le monde s'accorde pour dire que la réforme française des licenciements économiques souhaite sécuriser les employeurs, une partie seulement des commentateurs considère qu'elle apporte davantage de sécurité également aux salariés. Cette controverse montre d'ores et déjà le caractère contingent et ambigu de la notion de sécurisation, qui ne saurait avoir le même sens selon que l'on se place du côté de l'employeur ou du salarié.

Les deux réformes procédurales française et italienne visent en tout cas toutes les deux à accorder une souplesse supplémentaire en matière de licenciements économiques, en les rendant plus sûrs, plus faciles et plus prévisibles pour l'employeur. Pourtant, et c'est principalement ce que montre cette comparaison, la flexibilisation des licenciements économiques n'emprunte pas les mêmes chemins juridiques en France et en Italie. La place du juge dans les nouveaux dispositifs focalise les différences.

\section{Des choix procéduraux opposés}

Les deux droits redessinent, directement ou indirectement, la place du juge dans les licenciements. En France, on souhaite expressément une moindre intervention de la justice, donc des juges, dans les licenciements économiques, et plusieurs outils sont concomitamment mobilisés à cette fin. La réforme italienne est, de son côté, centrée sur le problème de la réintégration des salariés licenciés.

\section{France : une méfiance envers les juges et le contentieux}

Plusieurs changements législatifs impactent, à divers degrés, la place du juge dans les licenciements économiques.

La méfiance du législateur vis-à-vis du juge revêt deux aspects différents, qu'il ne faut ni confondre ni assimiler. Tout d'abord, le nouveau droit cherche à sécuriser la procédure afin d'éviter les contentieux. L'idée défendue est que l'employeur, s'il est victime de trop de recours en justice lors d'un licenciement économique, pourrait hésiter à l'avenir à embaucher. Les nouvelles normes visent cependant également à réduire, dans une certaine mesure, les pouvoirs du juge dans le contrôle des procédures.

\section{Éviter les contentieux individuels}

Tout d'abord, afin de purger une partie du contentieux, les délais de prescription pour agir en justice sont raccourcis. Le nouvel article L. 1471-1 du Code du travail prévoit dorénavant que l'action en justice portant sur l'exécution ou la rupture du contrat se prescrit au terme de deux ans à compter du jour où celui qui l'initie a connu ou aurait dû connaitre les faits lui permettant d'exercer son droit. Pour les licenciements économiques, la prescription, encore plus courte, est d'un an. En l'espace de quelques années, les délais de contestation d'un licenciement seront donc passés de trente à cinq ans, puis à un ou deux ans suivant la nature de la rupture. La prescription pour les rappels de salaires est également abrégée : elle passe de cinq à trois ans (art. L. 3245-1). C'est cette dernière prescription qui, assez discrètement, risque d'avoir le plus d'impact en matière contentieuse, et de dissuader tout recours en justice lors d'un licenciement. En effet, bien souvent, un salarié n'ose demander des rappels d'heures non payées que lorsque le contrat est rompu. Il s'agit ainsi souvent d'une demande accessoire en complément de la demande principale relative, par exemple, à la contestation du motif réel et sérieux du licenciement. Raccourcir le délai de prescription des rappels de salaire de cinq à trois ans réduit ainsi les espoirs de gain en justice, et peut donc dissuader un contentieux prud'homal toujours long, coûteux, et incertain. Le raccourcissement des délais de prescription cherche donc, parfois à neutraliser et le plus souvent à dissuader les actions émanant des salariés devant les conseils de prud'hommes: pourquoi, en effet, aller en justice lorsque le gain éventuel à l'issue d'une procédure devient faible ? Ce nouveau régime devrait ainsi accélérer la baisse du contentieux devant les conseils de prud'hommes. 
Celle-ci se constate déjà depuis 2009: le nombre d'affaires nouvelles introduites devant les conseils de prud'hommes a diminué de $23 \%$ entre 2009 et 2012, passant de 228901 à 175714 (GuillonNEAU, SERVERIN, 2013, p. 7).

\section{Limiter les contrôles liés au plan de sauvegarde de l'emploi}

En second lieu, le juge du tribunal de grande instance (TGI) est dépossédé d'une large part de sa compétence, au profit du tribunal administratif. Derrière ce changement a priori anodin de compétence juridictionnelle se cache la volonté de lutter, sans les remettre en cause frontalement, contre deux anciennes jurisprudences issues des arrêts Samaritaine et Alefpa ( $c f$. infra). Avec cette remise en cause discrète, l'objectif est d'amoindrir le contrôle judiciaire des plans de sauvegarde de l'emploi (PSE).

Le TGI est traditionnellement chargé des litiges collectifs du travail. C'est donc lui qui avait à juger des contestations relatives aux plans de sauvegarde de l'emploi, au fond comme en référé. Le juge administratif est désormais compétent pour connaître du recours contre la décision de contrôle sur la procédure du licenciement économique collectif exercé en amont par l'administration (29).

Dorénavant, le contenu du PSE est établi suivant deux procédures possibles : soit par accord collectif majoritaire (préalable à l'information-consultation du comité d'entreprise prévue par l'article L. 123324-1); soit, à défaut, par un document unilatéral de l'employeur définitivement élaboré après la dernière réunion du comité d'entreprise (art. L. 1233-24-1 à L. 1233-24-4). L'autorité administrative ${ }^{(30)}$ doit être informée du déroulé de la procédure avant même l'information-consultation formelle du comité d'entreprise (art. L. 1233-57 et s.). Elle est chargée d'évaluer les PSE via l'une ou l'autre des procédures retenues et la décision qu'elle prendra sera, le cas échéant, attaquable devant les juridictions administratives (en lieu et place du TGI). Si cette évaluation administrative peut, de prime abord, sembler une solution pertinente, se rapprochant du défunt contrôle administratif des licenciements économiques ${ }^{(31)}, 1$ l'étendue du contrôle prévu dans le droit actuel fait dire à certains auteurs que la loi «modifie substantiellement la mission de l'administration, en la transformant en acteur de certification des PSE» (Guiomard, Serverin, 2013, p. 197). L'intervention de l'administration, déclinée aux articles

(29) Le TGI garde une compétence résiduelle sur les contentieux des accords et des conventions collectives. Il garde ainsi sa compétence dans les litiges relatifs aux accords de maintien de l'emploi, en cas d'application déloyale par l'employeur.

(30) Il s'agit ici de la Direction régionale des entreprises, de la concurrence, de la consommation, du travail et de l'emploi (Direccte).

(31) Un contrôle administratif des licenciements économiques a existé entre 1975 et 1986.
L. 1233-57-1 à L. 1233-57-8, consiste essentiellement, selon les termes de l'article L. 1233-57-1, à «valider l'accord», ou, en l'absence d'accord majoritaire, à «homologuer le document unilatéral». Concernant la validation des accords, l'autorité administrative n'effectue que des vérifications de pure conformité formelle (art. L. 1233-57-2), dans un délai bref de quinze jours. En l'absence d'accord, l'homologation du document unilatéral n'est guère plus approfondie (art. L. 1233-57-3); le délai est certes plus long, mais demeure intrinsèquement court (vingt et un jours).

La décision administrative peut être contestée devant les tribunaux administratifs. Les juges administratifs n'auront qu'à contrôler le respect par l'autorité administrative des articles L. 1233-57-2 et L. 1233-57-3, c'est-à-dire à vérifier le bon déroulé d'une opération de certification. Une incertitude, sur laquelle nous reviendrons, demeure sur l'étendue exacte de ce contrôle.

Cette modification procédurale vise donc à éviter, en grande partie, les risques judiciaires liés aux jurisprudences Samaritaine et Alefpa dont voici les grands principes. L'arrêt Samaritaine ${ }^{(32)}$ affirmait qu'en cas de nullité du plan social ${ }^{(33)}$ constatée par un TGI, les licenciements effectués en application de ce plan pouvaient également être annulés. En conséquence, la Cour de cassation enjoignait aux juges de TGI de contrôler de manière approfondie la suffisance du plan social, pour déterminer, ou non, sa validité. Et en cas d'insuffisance, la nullité pouvait s'étendre à tous les actes subséquents, en particulier le licenciement prononcé par l'employeur (34). Lors de l'affaire Samaritaine, le TGI constata la nullité du plan social. Le Conseil de prud'hommes, ultérieurement saisi individuellement par des salariés, ne statua pas, quant à lui, sur la nullité du plan social, mais sur les conséquences de cette nullité sur les licenciements. Dès lors, on s'est demandé ce que pouvait faire un salarié estimant nul le plan de sauvegarde de l'emploi, et donc son licenciement, en l'absence d'action en nullité préalablement intentée devant le TGI. La Cour de cassation a été au bout de sa logique en indiquant, dans l'arrêt Alefpa (35), que «les salariés licenciés pour motif économique ont un droit propre à faire valoir que leur licenciement est nul au regard des dispositions de l'article L. 321-4-1». Ainsi, bien que le comité d'entreprise eût vocation à défendre les intérêts collectifs des salariés lors de licenciements collectifs, et notamment à soulever l'éventuelle insuffisance d'un plan

(32) Cour de cassation, chambre sociale, 13 février 1997, «Samaritaine», Bulletin civil, Ve partie, $n^{\circ} 64$.

(33) Ancienne dénomination du plan de sauvegarde de l'emploi.

(34) En droit, la nullité est en principe rétroactive : un licenciement nul n'est censé n'avoir jamais existé, entraînant la réintégration du salarié.

(35) Cour de cassation, chambre sociale, 30 mars 1999 , «Alefpa», Bulletin civil, Ve partie, $\mathrm{n}^{\circ} 144$. 
de sauvegarde de l'emploi, cela ne privait pas le salarié d'un intérêt personnel et légitime à contester, devant le Conseil de prud'hommes, son licenciement sur le fondement de l'insuffisance du PSE.

La nouvelle procédure a des conséquences sur les contentieux individuels et collectifs relatifs au PSE.

Pour ce qui est des contentieux individuels, elle empêche en grande partie la contestation individuelle d'un PSE devant le Conseil de prud'hommes avec demande de nullité du licenciement, le plan étant dorénavant préalablement contrôlé et validé par l'administration. Une procédure de licenciement économique définitivement validée ne sera plus contestable par le salarié devant le Conseil de prud'hommes, ce qui rend la procédure plus sûre pour l'employeur.

Les conséquences sont encore plus importantes pour les litiges collectifs car désormais toute contestation en référé avant le contrôle de la procédure par l'administration est impossible. Auparavant, en cas d'insuffisance manifeste du plan de sauvegarde de l'emploi, les instances représentatives du personnel saisissaient sans délai le TGI, pendant la procédure de licenciement collectif, afin qu'il la suspende. Ces actions avaient mécaniquement comme effet de repousser sine die les projets de licenciement, parfois pendant des années dans le cas d'un employeur violant à répétition les obligations légales, notamment en matière d'information et de consultation des comités d'entreprise ou de contenu insuffisant du plan de sauvegarde de l'emploi. Désormais, les actions en contestation de la procédure de licenciement économique collectif ne peuvent plus avoir lieu qu'une fois le contrôle réalisé par l'administration. L'objectif est d'éviter des contentieux parfois longs, comme ceux ayant opposé des années durant les syndicats et les instances représentatives du personnel à la société Goodyear ${ }^{(36)}$ sur les diverses versions du plan de sauvegarde de l'emploi.

Ces modifications démontrent bien que l'on peut modifier le droit du licenciement économique sans changer la définition du motif économique, ni même expressément censurer les jurisprudences Samaritaine et Alefpa, simplement en cherchant à réduire le contentieux, voire à le rendre impossible en référé.

(36) Après sept années de conflit judiciaire, le litige entre certains syndicats et la direction de l'entreprise Goodyear s'est clos en janvier 2014 avec la signature par les syndicats d'un protocole de fin de conflit acceptant la dernière version du PSE proposée par l'employeur. Voir sur cette affaire la Commission d'enquête parlementaire "relative aux causes du projet de fermeture de l'usine Goodyear d'Amiens-Nord et à ses conséquences économiques, sociales et environnementales et aux enseignements liés au caractère représentatif qu'on peut tirer de ce cas», sur le site de l'Assemblée nationale : http://www. assemblee-nationale.fr/14/dossiers/fermeture_usine_goodyear. asp; page consultée le 16 juin 2015.
Selon certains auteurs, dans ce nouveau droit du licenciement économique, le juge est considéré comme un "gêneur» (GREvy, HENRIOT, 2013), alors même que sa première fonction, primordiale dans un État de droit, est de trancher les litiges, donc de résoudre les conflits et de restaurer, par le droit, une certaine forme de paix sociale.

\section{Italie : une réaffirmation - provisoire - du rôle du juge}

En Italie, la réforme des licenciements économiques collectifs s'est construite contre l'article 18 du statut des travailleurs. Ces modifications, dans leur version de 2012, donnent plus de pouvoirs au juge dans l'évaluation du caractère légitime des licenciements économiques. Pour comprendre la réforme, il faut revenir sur le droit antérieur à 2012.

\section{L'ancienne réglementation (1970-2012)}

Lorsque le licenciement s'avérait injustifié, les conséquences indemnitaires différaient en fonction de la taille de l'entreprise. Le législateur prévoyait deux «protections» (tutela) possibles pour le salarié licencié sans juste motif : la protection réelle et la protection obligatoire (tutela reale / tutela obbligatoria), qui pouvaient, selon les hypothèses, permettre un dédommagement, une réintégration ou une réembauche. Le régime indemnitaire a été profondément modifié par la réforme Fornero.

Détaillée à l'article 18 du statut des travailleurs de 1970 , la protection réelle posait le principe, en cas de licenciement illégitime, d'une réintégration $\mathrm{du}$ salarié à son poste (reintegrazione nel posto di lavoro); elle prévoyait également la condamnation de l'employeur à payer des dommages et intérêts (risarcimento del danno) d'une somme au moins égale à cinq mois de salaire, éventuellement majorée en cas de comportement fautif de l'employeur, et par tout autre préjudice prouvé. L'employeur devait en outre payer les salaires correspondant à la période illégitime de rupture du contrat. Le salarié, qui ne souhaitait pas réintégrer son poste, avait la possibilité de demander des dommages et intérêts, au moins équivalents à quinze mois de salaire. Cette protection réelle constituait une forte garantie juridique pour le salarié abusivement licencié, et un coût économique certain pour l'employeur. Le régime de la protection réelle s'appliquait dans les trois hypothèses suivantes ${ }^{(37)}$ : si l'employeur employait plus de quinze salariés (ou plus de cinq dans une entreprise agricole) dans chaque unité productive ou dans chaque bureau ou établissement (ufficio); si l'employeur employait plus de quinze salariés (ou plus de cinq dans une entreprise agricole) dans la même commune, même s'ils étaient répartis au sein

(37) Article 18, alinéa 1 , loi $n^{\circ} 300 / 1970$. Ce point n’est pas changé dans la réforme de 2012, mais est relégué en fin d'article. 
de plusieurs unités productives ou établissements; si l'employeur employait plus de soixante salariés.

Le régime de la protection obligatoire, qui concerne a contrario les hypothèses non couvertes par celui de la protection réelle n'est pas modifié.

Protection minimale, de droit commun, contre les licenciements injustifiés, la protection obligatoire est prévue à l'article 8 de la loi n ${ }^{\circ} 604 / 1966$ modifié par l'article 2 de la loi $n^{\circ} 108 / 1990$. En cas de jugement déclarant illégitime le licenciement, l'employeur est condamné à réembaucher (riassumere) le salarié sous trois jours, ou bien, s'il le juge plus souhaitable, à le dédommager de son préjudice en lui versant une indemnité comprise entre deux mois et demi et six mois de salaire, calculée en fonction du nombre de salariés dans l'entreprise, de l'ancienneté du salarié licencié et des éventuelles fautes commises. L'employeur a ainsi le choix entre le dédommagement et la réembauche. Le salarié peut également, le cas échéant, refuser d'être réembauché et préférer une indemnisation. Il n'a par contre pas la possibilité de solliciter le réemploi à la place d'un dédommagement. La protection obligatoire est en ce sens plus faible pour le salarié qui ne peut s'opposer à la rupture de son contrat si l'employeur ne veut pas le réembaucher.

\section{La réforme Fornero (2012-2014)}

La réforme de 2012 modifie en profondeur l'article $18 \mathrm{du}$ statut des travailleurs et diminue la «protection réelle» du salarié en cas de licenciement. Le régime de réintégration ou d'indemnisation dépend de la nature du licenciement (38). Par exemple, en cas de licenciement discriminatoire, une possibilité de réintégration est maintenue. Concernant les licenciements économiques, le nouveau régime juridique n'est guère simple. En cas de licenciement économique individuel (giustificato motivo oggettivo ${ }^{(39)}$ ) jugé illégitime, l'employeur n'est en principe plus tenu de réintégrer le salarié, mais devra lui verser une indemnité comprise entre quinze et vingt-quatre mois de salaire. Le législateur a donc bien mis fin à la protection réelle du salarié que constituait sa réintégration en cas de licenciement économique dénué de «cause réelle et sérieuse» (pour reprendre, peutêtre improprement, les catégorisations françaises). Ce faisant, le législateur va dans le sens d'anciennes

(38) L'article 18 du statut des travailleurs se voit adjoindre pas moins de sept alinéas supplémentaires distinguant les diverses modalités de sanctions suivant les types de licenciements illégitimes. Voir DE LuCA (2013).

(39) Comme nous l'avons vu, le licenciement pour juste motif objectif n'est toutefois pas totalement assimilable au licenciement pour motif économique français. Sur ce point, V. Dalmasso, 2009, p. 32 et s. revendications patronales ${ }^{(40)}$ qui militaient pour la remise en cause de ce régime de réintégration.

Cependant, s'il est avéré que le fait objectif ayant motivé le licenciement est non seulement illégitime mais aussi manifestement inexistant ${ }^{(41)}$, le juge peut quand même ordonner la réintégration du salarié.

Le régime des licenciements économiques collectifs est également concerné par la réforme. En cas de licenciement "inefficace ${ }^{(42)}$ » d'un point de vue procédural, c'est-à-dire si l'employeur n'a pas respecté, par exemple, les règles encadrant la procédure de licenciements collectifs, le juge peut condamner l'employeur à verser au salarié une indemnité d'un montant compris entre six et douze mois de salaire.

C'est sur le contrôle des motifs de licenciement économique individuel que la réforme est la plus intéressante. Tout en flexibilisant expressément les ruptures, en supprimant le principe de la réintégration systématique au profit de celui du dédommagement, la nouvelle loi laisse au juge une marge de manœuvre inédite : si celui-ci estime que le licenciement est manifestement inexistant ou infondé, il garde la possibilité d'ordonner la réintégration, tandis que s'il le juge simplement illégitime, la sanction sera une indemnisation. Les juges ont ainsi acquis en 2012 un pouvoir large de modulation des sanctions à l'encontre des employeurs, modulation bien supérieure à ce qui existe en France. La flexibilité s'est donc construite en s'appuyant sur les juges. Comme nous le verrons, cette réforme n'a finalement été appliquée que pendant deux ans.

Un économiste italien indiquait, de manière un peu provocatrice, qu'en matière de relations de travail, la flexibilité ne suffisait jamais (BRUNO, 1989, p. 33). On le constate ici dans les discours juridiques français et italiens en matière de licenciement économique. La justification se fonde sur les sciences économiques: la flexibilité supprimerait des obstacles à l'embauche. Cependant, concrètement, elle prend des formes variées, voire contradictoires. Ainsi, nous sommes en présence de deux systèmes juridiques et économiques proches qui, avec la même intention juridique de flexibilisation du marché du travail, conduisent deux réformes

(40) L'ancien directeur général de la Cofindustria (syndicat des employeurs italiens), S. Parisi, écrivait ainsi en 2003 : «abolire l'art. 18 è un atto di civiltà» (abolir l'article 18 est une preuve de civilisation). V. «L'affondo di Parisi : l'obiettivo è abolire l'art. $18 »$, Corriere della sera, 12 giugnio 2003, p. 9.

(41) Le législateur crée de fait deux niveaux de licenciements économiques non justifiés, aux conséquences indemnitaires différentes. Comme nous le verrons, la différence entre les deux niveaux est largement laissée à l'appréciation du juge.

(42) Le terme italien "inefficace» ne peut se traduire par le mot français «inefficace» car le licenciement a eu lieu et produit un effet juridique (la rupture du contrat) sans réintégration du salarié. Aussi, il semble plus juste de traduire le terme par «irrégulier» (terminologie employée dans le droit français) ou, si l'on veut rester plus proche du mot italien, par «inopérant». 
opposées quant au rôle, et à l'utilité d'un acteur juridique central, à savoir le juge. Il ne s'agit pas ici de déterminer quel système est sur une bonne ou une mauvaise piste, mais plutôt de souligner, avant même toute étude sur la corrélation entre flexibilité et emploi, la contingence des déclinaisons juridiques du concept économique de flexibilité.

Ces réformes doivent être appréciées, autant que possible, à l'aune de leurs résultats. Il est encore trop tôt pour analyser pleinement l'application de ces normes. Il semble toutefois que les réformes italiennes comme françaises sont susceptibles de générer des effets secondaires non prévus par les législateurs - des sortes de "pochettes surprises», pour reprendre une expression employée par Gérard LyON-CAEN (2004), que nous pouvons tenter de découvrir.

\section{Des résultats incertains}

À deux visions juridiques différentes de la flexibilité correspondent deux sources de déconvenues différentes.

En Italie, la réforme de 2012 a été considérée comme un échec par le gouvernement Renzi. Les précisions jurisprudentielles devant être apportées au nouvel article 18 ont fait craindre une insécurité juridique pour les employeurs ainsi que le développement d'une jurisprudence trop protectrice des salariés. Fin 2014 et début 2015, le gouvernement Renzi a donc modifié en profondeur la procédure des licenciements économiques. En France, le risque d'échec existe aussi. Il n'est pas certain que le contentieux, notamment collectif, baisse; de même, il n'est pas sûr que les juridictions administratives soient plus clémentes envers l'employeur que les TGI, ce qui mettrait à mal le lien entre flexibilité et sécurité.

\section{Une nouvelle réforme en Italie}

Globalement, la réforme de 2012 n'a pas été bien accueillie par la doctrine juridique ${ }^{(43)}$. Alberto VALENTI (2012, pp. 243 et 248) note par exemple que cette réforme constituait une rupture par rapport à l'évolution antérieure du droit italien, qui souhaitait limiter les pouvoirs du juge dans les licenciements économiques. Il indique ainsi qu'un décret( ${ }^{(44)}$ de 2003 précisait que le contrôle judiciaire ne pouvait s'étendre jusqu'à l'examen des évaluations et des choix techniques, d'organisation ou de production. La jurisprudence de la Cour de cassation allait de toute façon dans ce sens, en prévoyant le caractère incontrôlable des choix de gestion de l'employeur

(43) Voir notamment, pour une vision critique de l'évolution de l'ensemble du droit du travail italien, Peruldi (2012).

(44) Décret législatif $n^{\circ} 276 / 2003$. (insindacabilità delle scelte imprenditoriali). $\mathrm{Au}$ contraire, la loi de 2012, en posant l'obligation de différencier les licenciements économiques illégitimes des licenciements économiques manifestement inexistants, requiert du juge un contrôle plus étroit et approfondi des choix de gestion de l'employeur.

La distinction entre licenciement au motif illégitime et licenciement au motif manifestement inexistant est selon la doctrine la source principale d'insécurité. Franco CARINCI (2012, p. 4) a même accusé le législateur d'avoir fabriqué, à la manière du docteur Frankenstein, un monstre juridique, très difficile à comprendre et à appliquer. Quelques décisions émanant des juges du fond ${ }^{(45)}$ ont commencé à décrire, de manière imparfaite, les hypothèses dans lesquelles le juge peut ordonner la réintégration (Marinelli, 2013, p. 2).

Face à ces incertitudes, le législateur, et le nouveau gouvernement dirigé par Matteo Renzi ont entrepris une nouvelle réforme des modes de contrôle et d'indemnisation des licenciements économiques par les juges. Cette loi du 10 décembre 2014(46), communément appelée "Jobs Act ${ }^{(47)}$ 》, a provoqué en décembre 2014 une grève générale, massive mais brève.

Son article 7c modifie le régime des sanctions des licenciements économiques pour les personnes embauchées à compter de la date de mise en application de la nouvelle loi. Cet article a pour but de «renforcer les opportunités d'emploi dans le monde du travail pour ceux qui sont en recherche d'emploi (48) ». Il prévoit notamment, pour les embauches à durée indéterminée, la création d'un nouveau type de contrat, le «contrat à protections croissantes ${ }^{(49)} \gg$, qui exclut, pour les licenciements économiques jugés illégitimes, la réintégration du salarié, au profit d'une indemnisation certaine et croissante avec l'ancienneté(50). Le décret législatif du 4 mars 2015 a précisé le contenu de cette loi ${ }^{(51)}$, et particulièrement le régime du nouveau contrat

(45) En particulier, le tribunal de Milan (20 novembre 2012) a considéré que le manquement à l'obligation de repêchage (reclassement) du salarié, de même que la violation de la procédure de licenciement (absence de procédure de conciliation) rendent le licenciement illégitime, donnant droit à des dommages et intérêts. En revanche, les juges ont estimé que la réintégration n'était pas possible, le motif du licenciement n'étant manifestement pas inexistant.

(46) Loi n 183/2014 du 10 décembre 2014.

(47) Sur cette loi, en langue française, voir ICHINO, MARTELLONI (2015) et BINI (2015).

(48) "Rafforzare le opportunità di ingresso nel mondo di lavoro da parte di coloro che sono in cerca di occupazione.» (Traduction de l'auteur dans le texte.)

(49) "Contratto a tutele crescente." (Traduction de l'auteur dans le texte.)

(50) «Escludendo per i licenziamenti economici la possibilità della reintegrazione del lavoratore nel posto di lavoro, prevedendo un indennizzo economico certo e crescente con l'anzianità di servizio. » (Traduction de l'auteur dans le texte.) (51) Décret-loi n 23/2015 du 4 mars 2015. 
de travail à «protections croissantes» (tutele crescenti) qui s'applique aux salariés embauchés en CDI à compter du 7 mars 2015. Afin de favoriser leur embauche, le législateur a mis au point un nouveau régime de licenciement. Pour les licenciements discriminatoires, ou réalisés oralement, la sanction reste la réintégration pour tous les salariés. Pour les licenciements pour motifs disciplinaires, la réintégration n'est possible que si l'on constate une inexistence du fait matériel contesté. Par contre, dans toutes les autres hypothèses, et notamment pour tous les licenciements économiques illégitimes, on parlera de licenciement injustifié. L'évolution est ici importante : la réintégration est impossible, et l'indemnisation va dépendre d'un barème imposé au juge. Celui-ci, fixé par le décret, est de deux mois de salaire par année d'ancienneté, avec un minimum de quatre mois versés, et un maximum de vingtquatre mois ${ }^{(52)}$.

Deux régimes d'indemnisation et de contrôle des licenciements économiques individuels coexistent donc désormais : pour les contrats à durée indéterminée signés avant le 7 mars 2015, le régime juridique demeure celui de la réforme Fornero de 2012 (avec un rôle important du juge dans le choix de la sanction et de l'indemnisation); pour les contrats signés après l'entrée en vigueur de la nouvelle loi, la réintégration ne sera plus possible en cas de licenciement économique illégitime, le juge ne pouvant ordonner que des dommages et intérêts financiers.

Cette dernière évolution du droit italien supprime donc en grande partie le principe de réintégration figurant dans l'article $18 \mathrm{du}$ statut des travailleurs en cas de licenciement économique. Ce faisant, elle limite le pouvoir du juge relatif à la détermination de la sanction applicable et à la fixation du montant des dommages et intérêts à octroyer. Son contrôle du caractère légitime du motif économique ne devrait cependant pas être affecté.

\section{En France, une résistance du contentieux à prévoir ?}

Il serait présomptueux d'affirmer pouvoir prévoir comment les acteurs (salariés, syndicats, employeurs, administrations, juges) vont s'approprier la nouvelle procédure de licenciement, et quelles en seront les conséquences, notamment contentieuses.

$\mathrm{Au}$ risque, assumé, de nous tromper, nous pressentons quand même que la loi de sécurisation de l'emploi pourrait générer quelques déceptions, pour les salariés mais surtout pour

(52) L'idée d'appliquer un barème aux indemnités de licenciement sans cause réelle et sérieuse est directement reprise en France en juillet 2015 dans la dernière version du projet de loi pour la croissance, l'activité et l'égalité des chances économiques ( $c f$. paragraphe suivant). les employeurs, et ne pas totalement réussir son entreprise de réduire la conflictualité judiciaire des licenciements.

Du côté des salariés, dont certains agissent parfois individuellement contre un licenciement économique, le risque principal, déjà largement à l'œuvre, est celui d'une acceptation du licenciement économique sans oser le contester devant les tribunaux. La réduction des délais de prescription et la validation préalable par l'administration de la procédure du licenciement économique sont susceptibles de dissuader le salarié d'agir en justice. Nous anticipons ainsi, au moins pour les licenciements économiques, une nouvelle baisse du contentieux devant les conseils de prud'hommes. À vrai dire, les chiffres sont d'ores et déjà en forte baisse : le nombre annuel des demandes d'indemnités liées à la rupture du contrat de travail pour motif économique est passé de 4875 en 2009 à 2497 en 2012 (soit - 49\%, contre - 23\% pour l'ensemble du contentieux prud'homal) ${ }^{(53)}$; et elles ne représentent plus que $1,4 \%$ des actions devant les conseils de prud'hommes ${ }^{(54)}$ (Guillonneau, Serverin, 2013, p. 8). Cette évolution se lit bien entendu différemment selon que l'on se place du côté du salarié ou de l'employeur. Du côté de l'employeur, on peut clairement soutenir que le licenciement économique a et aura à l'avenir peu de risques d'être contesté par le salarié. De ce point de vue, la sécurisation juridique du licenciement économique est acquise, et elle l'était d'ailleurs avant même la réforme de 2013. Du côté des salariés, cela veut-il dire que le licenciement économique est mieux accepté ? Il est difficile de répondre à cette question. Contentons-nous toutefois de signaler que le salarié renonçant à agir en justice risque d'éprouver une certaine frustration vis-à-vis de son employeur, du droit du licenciement, et des juges.

Malgré un risque contentieux individuel faible, le législateur est de nouveau intervenu pour tenter de «sécuriser» les licenciements. Faisant écho au droit italien, ce qui montre une nouvelle fois la forte interdépendance entre les deux droits, le

(53) Évolution en partie due au développement sur la période de la rupture conventionnelle homologuée, qui est en vigueur depuis 2008

(54) Cette forte baisse est délicate à interpréter. Elle résulte peut-être en partie du fort succès de la rupture conventionnelle homologuée, qui pourrait, dans un certain nombre de cas, se substituer à des licenciements économiques individuels. Un autre facteur de baisse, plus ancien, résulte dans le fait que, lors d'un licenciement économique, le salarié peut adhérer à un contrat de sécurisation professionnelle, lui conférant un certain nombre d'avantages en matière de recherche d'emploi. Si le salarié accepte ce contrat de sécurisation professionnelle, le licenciement économique est alors qualifié, un peu artificiellement, par le Code du travail, de rupture «d'un commun accord», pouvant laisser (improprement) penser que la contestation judiciaire du licenciement n'est plus possible. 
projet de loi pour la croissance, l'activité et l'égalité des chances économiques, définitivement adopté par l'Assemblée nationale le 9 juillet 2015, a introduit, au cours de sa dernière lecture une surprenante réforme du calcul des indemnités de licenciement sans cause réelle et sérieuse. Sous réserve de validation par le Conseil constitutionnel ${ }^{(55)}$, la loi prévoit, dans son article 266, une modification des articles L. 1235-3 et suivants $\mathrm{du}$ Code du travail. La nouvelle loi fixe, comme en Italie, un plafonnement des indemnités dues par l'employeur en cas de reconnaissance par le Conseil de prud'hommes du caractère injustifié du licenciement. Avant cette réforme, la loi ne définissait pas de montant maximum d'indemnisation : il appartenait au juge d'évaluer le préjudice subi par le salarié du fait de son licenciement sans cause réelle et sérieuse, en appliquant le principe général du droit à une réparation intégrale du préjudice. Il existait cependant, pour les salariés ayant plus de deux ans d'ancienneté et travaillant dans des entreprises de onze salariés et plus, une indemnisation minimale fixée à six mois de salaire brut.

La loi plafonne désormais les indemnités de licenciement sans cause réelle et sérieuse. Par exemple, pour les entreprises de moins de vingt salariés, elles sont équivalentes à de trois à douze mois de salaire selon l'ancienneté. Pour les salariés ayant moins de deux ans d'ancienneté, le maximum sera de trois ou quatre mois selon la taille de l'entreprise. L'objectif clairement affiché par le gouvernement est de limiter les frais pour les petites entreprises, et d'encourager le recrutement en contrat à durée indéterminée, même quand l'employeur n'est pas sûr de pouvoir garder longtemps son salarié. Dans les entreprises de plus de trois cents salariés, une indemnité maximale est également prévue. Elle sera de quatre, douze ou vingt-sept mois de salaire, selon l'ancienneté. Le plafonnement des indemnités de licenciement sans cause réelle et sérieuse répond à un double objectif : permettre aux employeurs de mieux connaître à l'avance le risque juridique lié à un licenciement et, en limitant les indemnités, de dissuader, lorsque la perspective de gain est trop faible, l'action en justice du salarié. Le licenciement économique étant peu contesté, cette réforme impactera certainement plus le contentieux des licenciements pour motifs personnels. Il n'est cependant pas acquis que ce nouveau cantonnement du rôle du juge soit conforme à la Constitution. Si cette réforme était validée, il n'est de toute façon pas certain qu'elle rende le droit plus prévisible et limite les contentieux : en effet, le juge recouvre ses pleins pouvoirs

(55) La loi a été définitivement votée le 9 juillet 2015, mais un recours devant le Conseil constitutionnel a été formé le 15 juillet 2015 par des députés et sénateurs issus du groupe les Républicains. NDLR : l'article a été bouclé fin juillet 2016 et ne peut donc tenir compte des développements ultérieurs. d'indemnisation (i.e. sans barème) dans certains cas, notamment quand le licenciement est jugé discriminatoire, en cas d'atteinte au droit de grève ou à une liberté fondamentale. Nul doute que les avocats spécialisés souléveront très fréquemment ces exceptions pour tenter d'obtenir une meilleure indemnisation de leur client... et provoqueront des contentieux inédits.

Cependant, selon la loi de sécurisation de l'emploi, c'étaient principalement les contentieux collectifs de travail en contestation des plans de sauvegarde de l'emploi qui étaient les plus problématiques. Comme nous l'avons déjà signalé, les contentieux en référé ont disparu, syndicats et instances de représentation du personnel ne pouvant saisir le tribunal administratif qu'une fois rendue la décision administrative relative à la procédure. Quelle sera alors la réaction des syndicats ou du comité d'entreprise ? Un premier bilan de la loi de sécurisation de l'emploi établi le 3 avril 2015 par le ministère du Travail (56) fait état d'une «baisse significative de la judiciarisation des plans sociaux» (p. 66 et suiv.). Le document annonce un taux de recours de $8 \%$ relatifs à des contestations des plans de sauvegarde de l'emploi devant le tribunal administratif, contre $25 \%$ devant le tribunal de grande instance avant la loi de sécurisation de l'emploi. En cas d'accord majoritaire le contentieux est encore inférieur, avec un taux de recours devant le tribunal administratif de $5 \%$. Ces chiffres semblent indiquer que l'objectif du législateur a été, au moins partiellement, atteint. Ils doivent toutefois être examinés avec prudence. Tout d'abord, la loi de sécurisation de l'emploi est encore récente: les 1142 plans de sauvegarde de l'emploi mis en place depuis son entrée en vigueur ne permettent pas encore de tirer des conclusions définitives sur l'appropriation de ce texte par les différents acteurs (employeurs, représentants de salariés et salariés). En outre, et surtout, la comparaison entre le contentieux devant le tribunal administratif et celui devant le tribunal de grande instance est délicate, car alors qu'auparavant il y avait deux types de contentieux devant les juges civils (en référé et au fond), il n'y en a dorénavant plus qu'un seul, au fond, devant le juge administratif. Pour être pertinent, il faudrait donc ne comparer que les contentieux au fond, mais la répartition entre fond et référé devant le TGI n'est pas précisée par le document cité.

Pour prévoir les risques éventuels de contentieux, il faut certainement, comme le fait le bilan

(56) Bilan de la loi de sécurisation de l'emploi du 14 juin 2013, ministère du Travail, de l'Emploi, de la Formation professionnelle et du Dialogue social. Disponible en ligne à l'adresse : http://travail-emploi.gouv.fr/IMG/pdf/ CONFERENCE_THEMATIQUE_DU_3_AVRIL_2015 Bilan_de_la_loi_de_securisation_de_1_emplōi.pdf; consultée le $17 \overline{\text { juin }} 20 \overline{1} 5$. 
du ministère du Travail, distinguer l'hypothèse de la présence d'un accord d'entreprise majoritaire de celle d'absence d'accord.

En cas d'absence d'accord avec, à la clef, l'élaboration d'un document unilatéral par l'employeur, les syndicats ne seront guère satisfaits du déroulé et du contenu de la procédure, en particulier lors de l'élaboration du PSE, et auront tendance à la contester devant les tribunaux administratifs. Dans cette hypothèse, on peut donc considérer que l'employeur ne cherchant pas à négocier efficacement (ou confronté à des syndicats ne souhaitant pas négocier...) prend un risque de voir son plan de restructuration contesté en justice. Le risque de contentieux est donc ici important du fait d'un climat social dégradé.

Le législateur a certainement considéré que, dans l'hypothèse d'un accord majoritaire, le contentieux serait faible, voire inexistant. Certes, tous les syndicats ayant signé l'accord seraient bien inconstants de contester en justice des éléments négociés. Cependant, les syndicats non signataires, qui peuvent représenter presque la moitié des effectifs salariés de l'entreprise, pourraient, eux, estimer qu'il est de leur devoir, vis-à-vis de leurs adhérents et de leurs électeurs, de contester la procédure. Un contentieux important n'est donc pas ici à exclure. Celui-ci dépendra aussi et surtout de la jurisprudence qui se mettra en place devant les juridictions administratives. En particulier, si les tribunaux administratifs, comme cela semble probable, souhaitent reprendre la jurisprudence antérieure développée par les TGI, il est à prévoir que le caractère suffisant du plan de sauvegarde de l'emploi sera mieux contrôlé par les juges administratifs que par l'administration: cela pourrait être une puissante incitation, pour les syndicats, à saisir systématiquement les tribunaux ${ }^{(57)}$. Dans cette hypothèse, l'idéal d'une déjudiciarisation des procédures des licenciements économiques aura fait long feu...

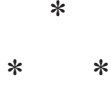

Une réforme italienne modifiée au bout de deux ans, et une réforme française susceptible de générer un contentieux en contestation des plans de sauvegarde des emplois devant les tribunaux administratifs : ces deux exemples montrent la difficulté de transcrire, en droit, la volonté de flexibilisation et de sécurisation des licenciements économiques. Le but de cette contribution n'était aucunement de juger une entreprise des plus complexes, voire téméraire, menée parfois courageusement par les législateurs et les partenaires sociaux, mais de souligner la difficulté même de concevoir, en droit, ce qu'est une législation portant davantage de flexibilité ou de souplesse en matière de licenciement économique. La contingence juridique de cette notion devrait donc inciter à la plus grande prudence. Avant toute réforme, il faudrait pouvoir tirer un bilan d'au moins vingt ans de renforcement de la flexibilité dans les relations de travail. Si celle-ci a effectivement rogné les protections dont bénéficiaient les salariés en CDI notamment en matière de rupture des contrats de travail, a-t-elle vraiment permis d'inciter à embaucher davantage ? Si des économistes ont d'ores et déjà remis en cause ce lien (PuCCI, VAlentin, 2008), l'idée d'une flexibilisation du droit du travail pour réduire le chômage demeure, dans ces dernières réformes, le fil conducteur des législateurs. Cependant, la difficulté, voire l'impossibilité de concevoir clairement en droit ce qu'est une législation flexible est à elle seule de nature à remettre en cause ce lien mécanique, «cette évidence scientifique», entre flexibilisation des ruptures et levée des freins à l'embauche.
(57) Le projet de loi «pour la croissance, l'activité et l'égalité des chances économiques» a peut-être anticipé cette hausse possible du contentieux. L'article 292 du texte adopté le 9 juillet 2015 à l'Assemblée nationale vise à limiter, dans certaines hypothèses, les effets d'une annulation par un tribunal administratif d'une décision de validation du PSE par l'administration: «En cas d'annulation d'une décision de validation mentionnée à l'article L. 1233-57-2 ou d'homologation mentionnée à l'article L. 1233-57-3 en raison d'une insuffisance de motivation, l'autorité administrative prend une nouvelle décision suffisamment motivée dans un délai de quinze jours à compter de la notification du jugement à l'administration. Cette décision est portée par l'employeur à la connaissance des salariés licenciés à la suite de la première décision de validation ou d'homologation, par tout moyen permettant de conférer une date certaine à cette information. Dès lors que l'autorité administrative a édicté cette nouvelle décision, l'annulation pour le seul motif d'insuffisance de motivation de la première décision de l'autorité administrative est sans incidence sur la validité du licenciement et ne donne lieu ni à réintégration, ni au versement d'une indemnité à la charge de l'employeur.» 


\section{Bibliographie}

BONNEChÈRE M. (2013), «Pour un autre modèle», in Couturier G., Akandji-Kombé J.-F. (dir.), Compétitivité des entreprises et sécurisation de l'emploi : le passage de l'accord à la loi, Paris, IRJS éd., pp. 145-152.

BINI S. (2015), «Les licenciements disciplinaires après le Job's act», Revue de droit du travail, n 6, pp. 420-425.

BRuno S. (1989), «La flexibilité, un concept contingent», in Maruani M., Reynaud E., Romani C., La flexibilité en Italie: débats sur l'emploi, Paris, Syros Alternatives, pp. 33-50.

CARINCI F. (2012), «Complimenti, dottor Frankenstein : il disegno di legge governativo in materia di riforma del mercato del lavoro », Il lavoro nella giurisprudenza, $\mathrm{n}^{\circ} 6$, pp. 1-39.

Couturier G. (2013), «Un nouveau droit des (grands) licenciements collectifs pour motif économique», Droit social, $\mathrm{n}^{\circ} 10$, pp. 814-826.

Dalmasso R. (2009), Essai sur le concept de licenciement économique, étude comparée des droits français et italien, Paris, LGDJ-Lextenso éd.

DAviD R. (1950), Traité élémentaire de droit civil comparé, introduction à l'étude des droits étrangers et à la méthode comparative, Paris, LGDJ.

De Luca M. (2013), «Riforma della tutela reale contro i licenziamenti al tempo delle larghe intese : riflessioni su un compromesso necessario», Rivista italiana di diritto del lavoro, $\mathrm{n}^{\circ} 1$, pp. 3-38.

FABre A. (2013), «Le licenciement économique et l'objectif de sécurisation», Revue de droit du travail, $\mathrm{n}^{\circ} 3$, pp. 184-191.

Foucher A. (2013), «La France entre démocratie politique et démocratie sociale: l'héritage d'une nation stato-centrée», in Couturier G., Akandji-Kombé J.-F. (dir.), Compétitivité des entreprises et sécurisation de l'emploi : le passage de l'accord à la loi, Paris, IRJS éd., pp. 89-95.

GÉA F. (2013), «Le droit du licenciement économique à l'épreuve de la sécurisation de l'emploi», Droit social, $\mathrm{n}^{\circ} 3$, pp. 210-222.

Grévy M., Henriot P. (2013), «Le juge, ce gêneur», Revue de droit du travail, $\mathrm{n}^{\circ} 3$, pp. 173-178.

Guillonneau M., Serverin É. (2013), L'activité des conseils de prud'hommes de 2004 à 2012 : continuité et changements, Paris, Ministère de la Justice, Direction des Affaires civiles et du Sceau, Pôle évaluations de la justice civile.

Guiomard F., Serverin É. (2013), «Les voies de contestation des décisions unilatérales des employeurs, à l'horizon de la loi sur la sécurisation de l'emploi», Revue de droit du travail, $\mathrm{n}^{\circ} 3$, pp. 197-201.
Ichino P., Martelloni F. (2015), «Le "Jobs Act" italien : quelles inspirations ?», Revue de droit du travail, $\mathrm{n}^{\circ} 5$, pp. 299-308.

Jolivet G. (2013), «La loi de sécurisation de l'emploi : de la flexisécurité à la pseudo-sécurité ?», JCPS, n 44-45, pp. 1-6.

LARDY S. (2013), «Accord national interprofessionnel du 11 janvier 2013 : dérégulation du droit du travail et logique sacrificielle», in Couturier G., Akandji-Kombé J.-F. (dir.), Compétitivité des entreprises et sécurisation de l'emploi : le passage de l'accord à la loi, Paris, IRJS éd., pp. 101-104.

Lyon-CAen G. (2004), «Les pochettes surprises de la chambre sociale, l'arrêt Aventis Pharma», Semaine sociale Lamy, $\mathrm{n}^{\circ} 1154$, pp. 6-11.

MARINELli F. (2013), «Il licenziamento per giustificato motivo oggettivo un anno dopo la riforma Fornero», Bollettinoadapt.it, 10 juillet, disponible en ligne à l'adresse : http://www.bollettinoadapt.it/old/files/ document/22538marinelli_10_7_1.pdf; consulté le 26 août 2015.

Perulli A. (2012), «Il diritto del lavoro tra libertà e sicurezza», Rivista italiana di diritto del lavoro, $\mathrm{n}^{\circ} 2$, pp. 247-288.

Pucci M., Valentin J. (2008), «Flexibiliser l'emploi pour réduire le chômage, une évidence scientifique ?», Connaissance de l'emploi, $\mathrm{n}^{\circ}$ 50, Noisy-le-Grand, Centre d'études de l'emploi.

Scognamiglio R. (2013), «Commento alla legge 28 giugno 2012, n.92 sulla riforma del diritto del lavoro», Rivista italiana del diritto del lavoro, $\mathrm{n}^{\circ} 4$, pp. 783-826.

Seigne A., Pirat E. (2013), «La CFDT signe un accord pour l'emploi », CFDT magazine, février, pp. 12-17.

SPEziale V. (2012), «La riforma del licenziamento individuale tra diritto ed economia», Rivista italiana di diritto del lavoro, $\mathrm{n}^{\circ} 3$, pp. 521-566.

TARASEwicz Y. (2013), «À propos de la nouvelle procédure de licenciement collectif pour motif économique», Semaine sociale Lamy, $\mathrm{n}^{\circ}$ 1592, pp. 61-64.

VAlenti A. (2012), «Le ragioni del licenziamento per motivi economici in tempo di crisi. Il "regolare funzionamento" dell'organizzazione del lavoro : ipotesi di ricerca», Ianus, n ${ }^{\circ}$ 7, pp. 239-264.

VIDIRI G. (2012), «La riforma Fornero : la (in)certezza del diritto e le tutele differenziate del licenziamento illegittimo», Rivista italiana di diritto del lavoro, $\mathrm{n}^{\circ} 4$, pp. 617-650.

ZWEIGERT K., KöTZ H. (1998), Introduction to comparative law, translated from the German by Tony Weir, $3^{\text {rd }}$ revised edition, Oxford, Clarendon Press. 